\title{
Serum miRNA-142 and BMP-2 are markers of recovery following hip replacement surgery for femoral neck fracture
}

\author{
HAOYUAN GAO $^{1}$ and XUYOU WANG ${ }^{2}$ \\ ${ }^{1}$ Department of Orthopedics, The Fourth Hospital of Jinan, Jinan, Shandong 250021; \\ ${ }^{2}$ Department of Laboratory Medicine, Wulian People's Hospital, Shandong 262300, P.R. China
}

Received August 28, 2019; Accepted June 17, 2020

DOI: $10.3892 / e t m .2020 .9235$

\begin{abstract}
The present study aimed to investigate the changes in miRNA-142 and bone morphogenetic protein-2 (BMP-2) expression before and after hip replacement surgery, and to determine their association with receptor activator of $N F-\kappa B$ ligand (RANKL) and osteoprotegerin (OPG). For this purpose, 142 cases of hip arthroplasty in patients with femoral neck fracture were selected as the research group, and 50 cases of healthy individuals who underwent a physical examination during the same time period were selected the control group. Serum miR-142 and BMP-2 levels were measured by RT-qPCR before and after surgery in the research group and the control group. Serum RANKL and OPG levels were detected before and after surgery by enzyme-linked immunosorbent assay (ELISA). The levels of serum miR-142 and BMP-2 in the research group were significantly lower than those in the control group. At 1 month after surgery, the levels of serum miR-142 and BMP-2 in the research group were significantly higher than those before surgery, and at 6 months after surgery, and they were higher than those in the control group. Pearson's correlation analysis revealed that the serum levels of miR-142 positively correlated with the BMP-2 levels before surgery, at 1 month after surgery, and at 6 months after surgery in the research group. The results of ROC curve analysis revealed that the AUC values of serum miR-142 and BMP-2 were 0.911 and 0.861 , respectively. At 1 month after surgery, the levels of serum miR-142 and BMP-2 in group A (patients with a good or excellent recovery) were significantly higher than those in group B (patients with a fair or poor recovery). The levels of serum RANKL and OPG in the research group significantly increased at 1 month after surgery. The serum levels of miR-142 and BMP-2 positively correlated
\end{abstract}

Correspondence to: Dr Xuyou Wang, Department of Laboratory Medicine, Wulian People's Hospital, 50 Limin Road, Rizhao, Shandong 262300, P.R. China

E-mail:w5lyyb@163.com

Key words: femoral neck fracture, hip replacement, miR-142, bone morphogenetic protein 2 , receptor activator of nuclear factor- $\kappa \mathrm{B}$ ligand, osteoprotegerin with those of RANKL and OPG before surgery and at 1 month after surgery. On the whole, the findings of the present study indicate that pre-operative serum miR-142 and BMP-2 levels are valuable for evaluating the post-operative recovery of patients with femoral neck fracture undergoing hip replacement surgery. In addition, in the present study, at 1 month after surgery, the levels of both miR-142 and BMP-2 were related to the recovery of the patients, and positively correlated with the RANKL and OPG levels.

\section{Introduction}

Hip fracture is an international public health concern. There are approximately 1.5 million cases of hip fracture worldwide each year. By the year 2050, it is estimated that there will be 3.9 million hip fractures worldwide and $>700,000$ cases in the United States (1). Femoral neck fracture is one of the most common types of hip fracture. Surgical treatment is usually required clinically. The purpose of surgical treatment is to immediately relieve pain, accelerate recovery and reduce the occurrence rate of surgical complications $(2,3)$. Currently, hip replacement surgery is widely used for the treatment of severe hip injury, and it is a very safe, reliable and effective surgical method. The 10 -year survival rate of patients with the implant is $>99 \%$ (4). Hip replacement can successfully restore the function and activity of the patient's joints, relieve pain and improve the quality of life; however, the recovery of hip joint function following surgery differs $(5,6)$.

MicroRNAs (miRNAs or miRs), a type of small non-coding RNA with approximately 22 nucleotides in length, participate in the regulation of gene expression and are a type of broad-spectrum RNA that coordinates biological processes (7). A series of miRNAs has been proven to regulate the osteogenic activity and bone formation of osteoblasts, and play an important role in regulating bone regeneration and remodeling (8). miR-142 has been proven to be overexpressed in various pathological environments, such as in atherosclerosis (9), inflammation of the small intestine (10) and gastric cancer (11). Bone morphogenetic protein 2 (BMP-2) is a hydrophobic acidic glycoprotein, which can induce the proliferation, differentiation and migration of interstitial cells, promote cartilage and bone formation, and can efficiently induce osteogenic activity (12). A number of studies have investigated the roles of miR-142 and BMP-2 in osteoarthritis. For example, in 
the study by Chen et al (13), miR-142-3p expression was shown to be significantly decreased in patients with osteoporosis, and was thus considered to be a biomarker for osteoporosis. Mi et al (14) also revealed that BMP-2 played an important role in fracture healing, and the loss of chondrocyte BMP-2 induced the formation of cartilaginous callus to be prolonged, thus affecting the process of fracture healing. The receptor activator of NF- $\kappa \mathrm{B}$ ligand (RANKL)/osteoprotegerin (OPG) system is the main signaling pathway regulating the differentiation and function of osteoblasts and osteoclasts. RANKL is a cytokine synthesized and secreted by osteoblasts, which can promote bone resorption $(15,16)$. Fractures can cause bone metabolism in the body. OPG originates from bone cells and is an important cytokine in bone metabolism. It can be combined with RANKL competitively to inhibit bone resorption and promote bone healing (17).

The present study analyzed the changes in serum miR-142 and BMP-2 in patients with femoral neck fracture before and after hip replacement surgery in order to examine their association with the post-operative recovery of the patients, as well as their association with RANKL and OPG.

\section{Materials and methods}

General data. From April 2013 to August 2016, 142 patients with femoral neck fracture undergoing total hip replacement at the Orthopedic Department of The No. 4 Hospital, Jinan, Shandong were selected as the research group. According to the American Society of Anesthesiologists (ASA) classification standard, there were 101 cases classified as class II and 41 as class III. The classification referred to the definition of the ASA grade published by Davenport (18). The inclusion criteria were as follows: Patients who met the diagnostic criteria for femoral neck fracture (19); patients with obvious hip pain, longitudinal percussion pain of the lower limbs, unilateral displaced femoral neck fracture shown by X-ray; patients who underwent total hip replacement surgery at the Orthopedic Department of The No. 4 Hospital, met ASA Class II-III standards, and patients without major organ dysfunction. The exclusion criteria were as follows: Patients who received hemiarthroplasty in the Orthopedic Department of The No. 4 Hospital; if the surgery was performed $>1$ week after the fracture; patients had a history of hip replacement, pathological fracture, lumbar disease and rheumatoid arthritis; patients with complications such as cerebrovascular diseases, mental diseases, peripheral neuropathy, connective tissue disease, endocrine metabolic disease, severe respiratory system disease, hematopoietic dysfunction, malignant tumor and infectious diseases; patients who used immunosuppressive, anti-inflammatory and hormone drugs over the past 1 month; patients died within 6 months after operation. In addition, 50 cases of healthy individuals who underwent a physical examination during the same time period were selected as the control group, including 23 males and 27 females, aged 43-76 years, with an average age of $60.1 \pm 8.6$ years. Patients without dysfunction of the heart, kidneys, liver and other important organs, and with a normal physical examination were selected for the present study. Patients understood the situation of the study and signed informed consent forms. This study did not violate any ethical regulations. The plan was submitted to the Ethics Committee of Orthopedic Department of The No. 4 Hospital for review and was implemented after obtaining approval.

Surgical treatment. The patients in the research group underwent total hip replacement surgery (20), and all patients underwent surgery within 1 week after sustaining injury. Prior to the surgery, a hip X-ray film was obtained to confirm the fracture status and evaluate the length, size and femoral offset reconstruction during the surgery. Skin preparation was performed for continuous epidural general anesthesia, the patients were placed in the lateral position and the lateral hip joint approach was performed. The attachment of $1 / 3$ of gluteus medius muscle was cut and the structures of the hip joint were exposed. The femoral head was removed following osteotomy. The acetabulum was removed and the acetabulum prosthesis was placed. The neck was removed and the distance of the femur was approximately $12 \mathrm{~cm}$. The femoral stem prosthesis was implanted and the lateral soft tissue of the hip joint was reconstructed. A routine cleaning inspection was performed, and the incision was closed after cleaning. Following surgery, $3 \mathrm{~g}$ of anti-infective cefotaxime sodium and $0.5 \mathrm{~g}$ of metronidazole were administered intravenously twice a day. Low molecular weight heparin (LMWH) was injected subcutaneously $8 \mathrm{~h}$ following surgery, and then once a day for 3 days.

Evaluation index. The Harris score (21) was applied 6 months after surgery to evaluate the recovery of the patients, including mobility (47 points), pain (44 points), deformity (4 points) and joint mobility surface (5 points). The total score was 100 points. The high score was closely related to better joint function and recovery. According to the Harris scale, the scoring was as follows: Excellent, $>90$ points; good, 80-90 points; fair, 70-79 points; and poor, $<70$ points.

Detection method. Following admission into the control group or prior to surgery, at 1 month after surgery and at 6 months after surgery in the research group, $5 \mathrm{ml}$ of venous blood was obtained from the patients on an empty stomach and placed in a vacuum blood collection vessel without anticoagulant, and centrifuged at $1,006.2 \mathrm{x}$ g for $10 \mathrm{~min}$ (with a centrifuge radius of $10 \mathrm{~cm}$ and a centrifuge temperature of $4^{\circ} \mathrm{C}$ ), and the separated upper serum was placed in EP tube for later use.

Serum total RNA was extracted according to the instructions provided with the TRIzol total RNA kit (Invitrogen; Thermo Fisher Scientific, Inc.). The concentration and purity of the RNA were determined using a 751GD UV-Vis spectrophotometer (Beijing Qianming Gene Technology Co., Ltd.). A total of $2 \mu \mathrm{l}$ of total RNA was used to prepare the cDNA according to the instructions provided with the PrimeScript RT Master Mix (RR036A; Takara Biotechnology Co., Ltd.). The synthesized cDNA sample was placed at $-20^{\circ} \mathrm{C}$ for later use. The primer sequences were designed and synthesized by Tiangen biotech (Beijing) Co., Ltd. and are listed in Table I. The PCR reaction included the following: $10 \mu \mathrm{l}$ of $2 \mathrm{X}$ miR qPCR Mix, $0.4 \mu \mathrm{l}$ of upstream primer, $0.4 \mu \mathrm{l}$ of downstream primer, $0.4 \mu \mathrm{l}$ of sample, the sample was supplemented to $20 \mu \mathrm{l}$ with dd water (RNase- and DNase-free). The PCR reaction conditions were as follows: Pre-denaturation at $90^{\circ} \mathrm{C}$ for $3 \mathrm{~min}$, denaturation at $90^{\circ} \mathrm{C}$ for $20 \mathrm{sec}$, annealing at $60^{\circ} \mathrm{C}$ for $20 \mathrm{sec}$, and extension at $72^{\circ} \mathrm{C}$ for $40 \mathrm{sec}$, for a total of 40 cycles. 
Table I. Sequences of primers used in the present study.

\begin{tabular}{lll}
\hline Gene & \multicolumn{1}{c}{ Upstream primer sequence } & Downstream primer sequence \\
\hline miR-142 & 5'-TGCAGGGCAGCAGAGGAGCTGCTGT-3' & 5'-ACTGAGGCTCTGGGCAGTCAGGACC-3' \\
U6 & 5'-CTCGCTTCGGCAGCACA-3' & 5'-AACGCTTCACGAATTTGCGT-3' \\
BMP-2 & 5'-AACCTGCAACAGCCAACT-3' & 5'-GCTCAGTGTAGCCCAGGAT-3' \\
GAPDH & 5'-GAAGGTCGGAGTCAACGG-3' & 5'-GCTCAGTGTAGCCCAGGAT-3' \\
\hline
\end{tabular}

Amplification data analysis was performed using 7500 fluorescence quantitative PCR (Applied Biosystems; Thermo Fisher Scientific, Inc.) manufacturer software, and the results were analyzed using the $2^{-\Delta \Delta \mathrm{Cq}}$ method (22).

The levels of serum BMP-2, RANKL and OPG were detected by enzyme-linked immunosorbent assay (ELISA) as previously described, and the detection was carried out with reference to the operating instructions of the human BMP-2, RANKL, OPG ELISA kits (all purchased from Zhenyu Biotechnology Co., Ltd.). The OD value of each well was detected at a $450 \mathrm{~nm}$ wavelength using an Elx-800 enzyme linked immunosorbent assay meter (BioTek Instruments, Inc.), and the BMP-2, RANKL, OPG levels were calculated. Serum alanine aminotransferase (ALT), aspartate aminotransferase (AST), creatinine (Cre) and blood glucose (Glu) levels were detected using a BS-280 automatic biochemical analyzer. White blood cell (WBC) count and platelet (PLT) count in peripheral blood were measured using a BC-5000 automatic blood cell analyzer. D-dimer, prothrombin time (PT), thrombin time (TT) and activated partial thromboplastin time (APTT) were detected using a C2000-A automatic coagulation analyzer. The instrument was purchased from Vedeng Medical Co., Ltd.

Statistical analysis. SPSS 20.0 (IBM Corp.) was used for statistical analysis, and GraphPad Prism 6 software was used to visualize the data. Measurement data are expressed as the mean \pm standard deviation (means $\pm \mathrm{SD}$ ). The t-test was used for comparisons between 2 groups of normally distributed data, and a paired t-test was used for comparisons before and after treatment. Non-normal distribution data are expressed as $\mathrm{M}(\mathrm{P} 25-\mathrm{P} 75)$. Count data are expressed as numbers and percentages [n $(\%)$ ], and the Chi-squared $\left(\chi^{2}\right)$ test was used for the comparisons of count data between groups. Repeated measurement analysis of variance was used for data comparisons at multiple time points, one-way analysis of variance was used for comparisons among multiple groups of mean. Pairwise comparisons were conducted using the Tukey's test. Pearson's correlation analysis was used for correlation analysis among continuous data, while Spearman's correlation analysis was used for grade data. ROC was used to plot the diagnostic value of preoperative serum miR-142 and BMP-2 levels for clinical efficacy. The P-value less than 0.05 was regarded as statistical significance.

\section{Results}

General data of the research group and control group. No significant differences were observed in sex, age, body mass index (BMI), smoking history, alcohol consumption history, alanine aminotransferase (ALT), aspartate aminotransferase (AST), white blood cell (WBC) count, D-dimer, prothrombin time (PT), thrombin time (TT), activated partial thromboplastin time (APTT), platelet (PLT) count, creatinine (Cre) and blood glucose (Glu) between the research group and control group $(\mathrm{P}>0.05)$ (Table II).

Serum miR-142 and BMP-2 levels in the research group and control group before and after surgery. The levels of serum miR-142 and BMP-2 in the research group before surgery were significantly lower than those in the control group $(\mathrm{P}<0.01)$. The levels of serum miR-142 and BMP-2 in the research group at 1 month after surgery were significantly higher than those before surgery and those at 6 months after surgery, and those in the research group were higher than those in the control group at 1 month after surgery $(\mathrm{P}<0.001)$. The levels of serum miR-142 and BMP-2 in the research group reached peak levels at 1 month after surgery, and no significant differences were found between the research group and control group at 6 months after surgery $(\mathrm{P}>0.05)$. The results of Pearson's correlation analysis revealed that the serum miR-142 level in the research group positively correlated with the BMP-2 level before surgery, at 1 month after surgery and at 6 months after surgery $(\mathrm{P}<0.001)$ (Fig. 1).

Value of pre-operative and post-operative levels of $\mathrm{miR}-142$ and BMP-2 in evaluating patient recovery. Following surgical treatment, the recovery in the research group was assessed as excellent in 60 cases, good in 55 cases, fair in 21 cases and poor in 6 cases. At 6 months after surgery, the Harris score was used to evaluate the recovery status of the patients. According to the recovery status, 60 patients exhibited excellent recovery and 55 patients exhibited good recover and were selected as Group A (115 cases); in addition, 21 patients exhibited a fair recovery and 6 patients exhibited a poor recovery and were selected as Group B (27 cases). The levels of serum miR-142 and BMP-2 in group A before treatment were significantly higher than those in group $\mathrm{B}(\mathrm{P}<0.01)$. ROC curve analysis of pre-operative serum miR-142 and BMP-2 levels for the evaluation of the recovery of patients following of hip arthroplasty was created. The AUC values of serum miR-142 and BMP-2 for evaluating the recovery of patients were 0.911 and 0.861 , respectively. Further evaluation of the association between post-operative miR-142 and BMP-2 levels with recovery revealed that the serum miR-142 and BMP-2 levels in group A were significantly higher than those in group B at 1 month after surgery. According to the recovery, the patients with poor, fair, good and excellent recovery were set to 1,2, 3 and 4 
Table II. General data of the study group and control group.

\begin{tabular}{|c|c|c|c|c|}
\hline Category & Study group $(n=142)$ & Control group $(n=50)$ & $\mathrm{t} / \chi^{2}$ value & P-value \\
\hline Sex & & & 0.810 & 0.368 \\
\hline Male & $55(38.73)$ & $23(46.00)$ & & \\
\hline Female & $87(61.27)$ & $27(54.00)$ & & \\
\hline Age (years) & $62.3 \pm 6.9$ & $60.1 \pm 8.6$ & 1.814 & 0.071 \\
\hline BMI $\left(\mathrm{kg} / \mathrm{m}^{2}\right)$ & $23.4 \pm 4.2$ & $22.8 \pm 4.9$ & 0.831 & 0.407 \\
\hline Smoking history & & & 0.364 & 0.546 \\
\hline Yes & $31(21.83)$ & $13(26.00)$ & & \\
\hline No & $111(78.17)$ & $37(74.00)$ & & \\
\hline Alcohol consumption history & & & 0.142 & 0.706 \\
\hline Yes & $35(24.65)$ & $11(22.00)$ & & \\
\hline No & $107(75.35)$ & $39(78.00)$ & & \\
\hline ALT (U/l) & $26.58(16.86-34.22)$ & $22.98(16.12-29.86)$ & 1.349 & 0.179 \\
\hline AST (U/l) & $17.29(12.26-21.90)$ & $16.26(11.98-24.48)$ & 0.169 & 0.866 \\
\hline WBC (x109/1) & $5.38 \pm 2.26$ & $5.26 \pm 2.48$ & 0.315 & 0.753 \\
\hline D-dimer $(\mu \mathrm{l} / \mathrm{ml})$ & $0.40(0.19-0.61)$ & $0.36(0.18-0.50)$ & 1.513 & 0.132 \\
\hline PT (sec) & $11.35 \pm 2.26$ & $10.61 \pm 3.53$ & 1.700 & 0.091 \\
\hline TT (sec) & $14.49 \pm 1.67$ & $14.25 \pm 1.37$ & 0.913 & 0.362 \\
\hline APTT (sec) & $33.51 \pm 5.92$ & $34.56 \pm 6.47$ & 1.053 & 0.294 \\
\hline PLT $\left(\times 10^{9} / 1\right)$ & $199.37(156.01-237.51)$ & $169.67(146.21-220.44)$ & 1.501 & 0.135 \\
\hline Cre $(\mu \mathrm{mol} / \mathrm{l})$ & $70.10(45.15-108.23)$ & $79.42(50.51-106.24)$ & 0.268 & 0.789 \\
\hline Glu (mmol/l) & $5.62 \pm 0.81$ & $5.51 \pm 1.02$ & 0.770 & 0.442 \\
\hline Surgery duration (min) & $95.4 \pm 17.6$ & - & - & - \\
\hline ASA classification & & & - & - \\
\hline II & $101(71.13)$ & - & & \\
\hline III & $41(28.87)$ & - & & \\
\hline
\end{tabular}

Data are presented as numbers and percentages [n (\%)] as means $\pm \mathrm{SD})$ or $\mathrm{M}(\mathrm{P} 25-\mathrm{P} 75)$.
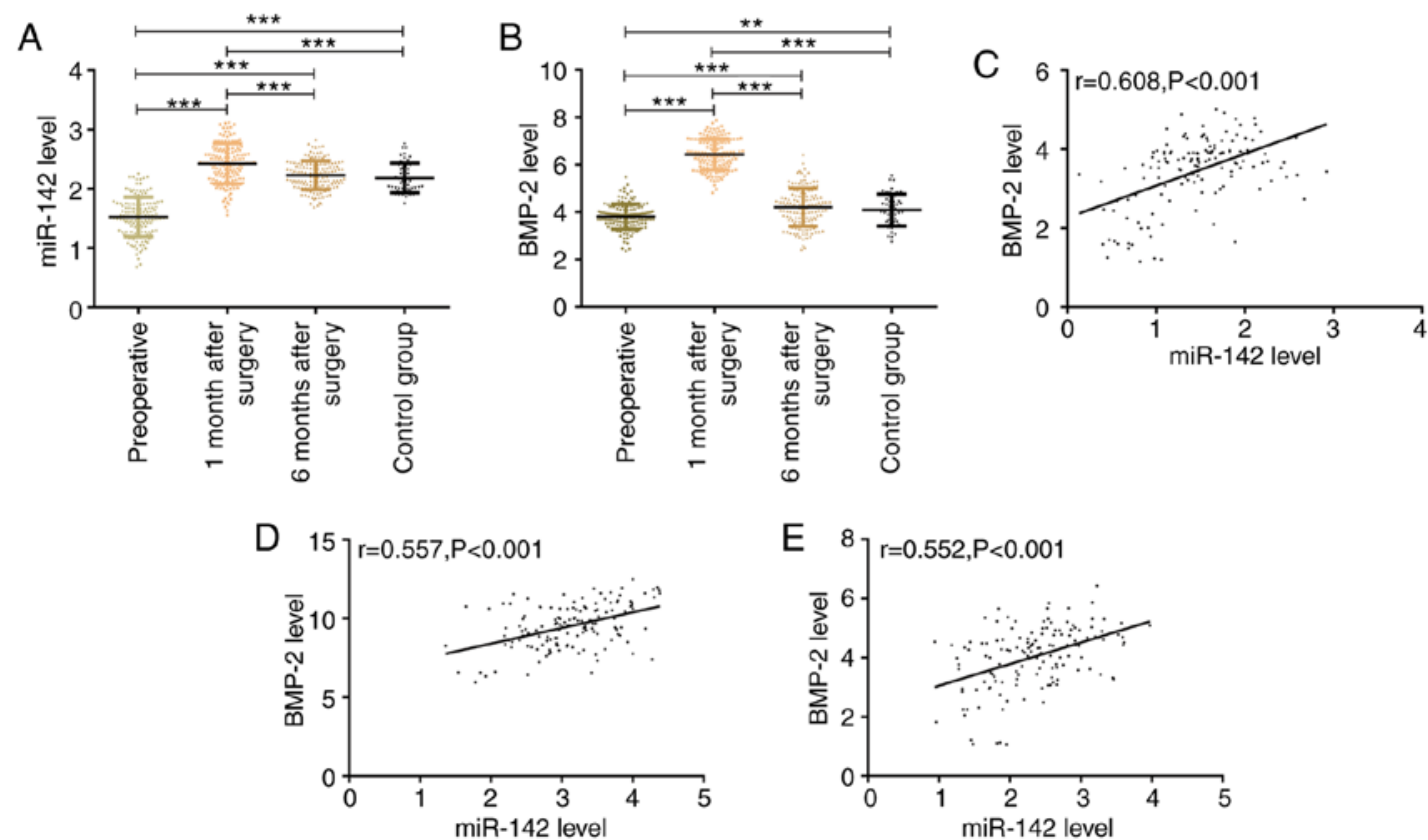

Figure 1. Serum miR-142 and BMP-2 levels before and after surgery in the research group and control group. Serum levels of (A) miR-142 and (B) BMP-2 in the research group before surgery, and at 1 month and 6 months after surgery, and in the control group. In the research group, serum miR-142 levels positively correlated with (C) BMP-2 levels before surgery, (D) at 1 month after surgery, and (E) at 6 months after surgery. ${ }^{* *} \mathrm{P}<0.01{ }^{* * * *} \mathrm{P}<0.001$. BMP-2, bone morphogenetic protein-2. 

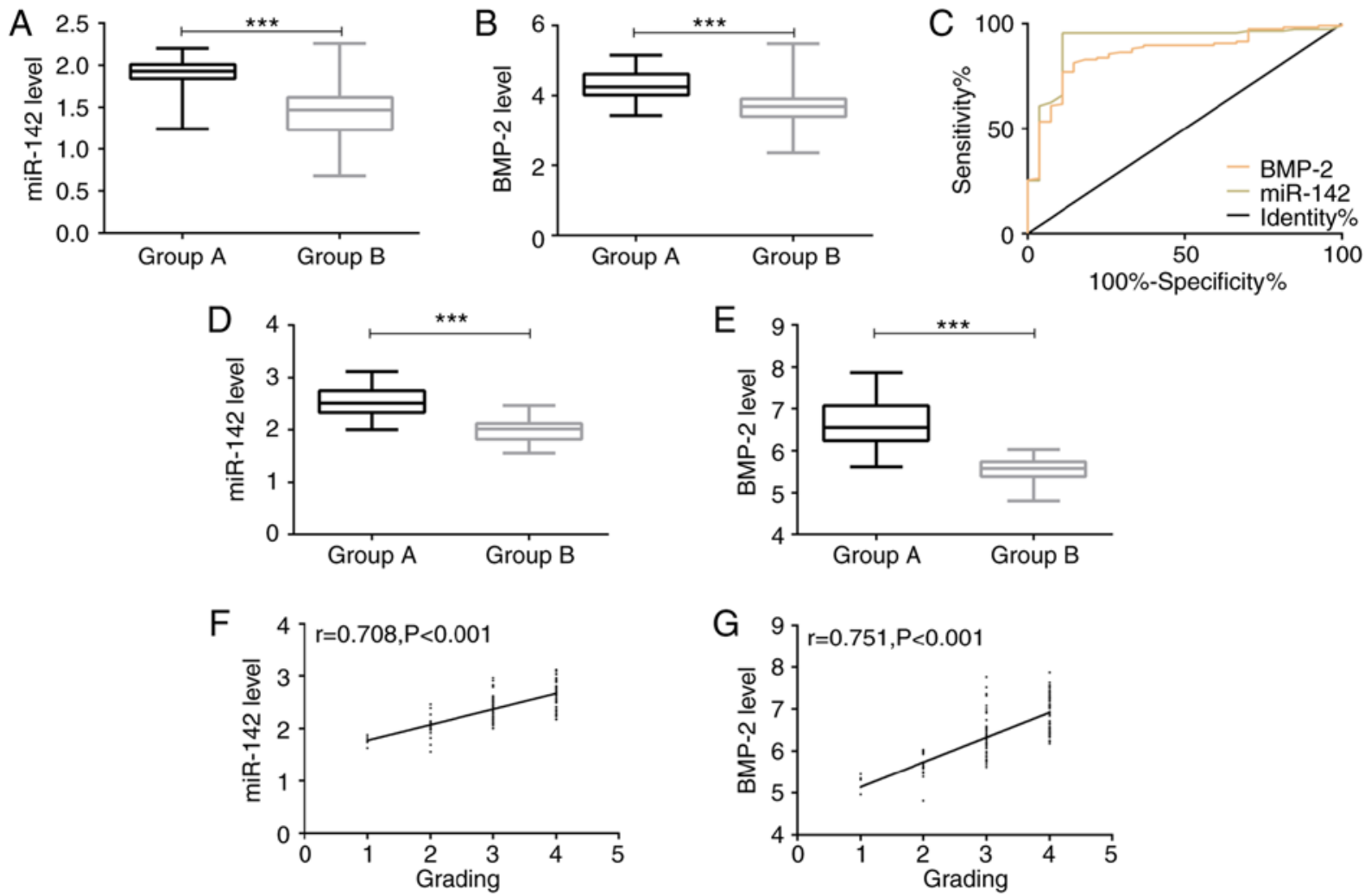

Figure 2. Diagnostic value of pre-operative miR-142 and BMP-2 levels on clinical efficacy in the research group. At 6 months after surgery, the Harris score was used to evaluate the recovery status of patients. According to the recovery status, 60 patients exhibited an excellent recovery and 55 patients exhibited a good recovery (group A; 115 cases); in addition, 21 patients exhibited a fair recovery and 6 patients exhibited a poor recovery (group B; 27 cases). The levels of serum (A) miR-142 and (B) BMP-2 in group A were significantly higher than those in group B before surgery. (C) ROC curve analysis of serum miR-142 and BMP-2 levels in the diagnosis and recovery of patients. At 1 month after surgery, the levels of serum (D) miR-142 and (E) BMP-2 in group A were significantly higher than those in group B. Serum (F) miR-142 and (G) BMP-2 levels positively correlated with the recovery effect. ${ }^{* * *} \mathrm{P}<0.001$. BMP-2, bone morphogenetic protein-2.

respectively. According to Spearman's correlation coefficient, serum miR-142 and BMP-2 levels positively correlated with recovery effect (Fig. 2 and Table III).

Serum RANKL and OPG levels before and after surgery in the research group. The serum RANKL levels in the research group were $6.19 \pm 1.44$ and $11.15 \pm 2.36 \mathrm{ng} / \mathrm{ml}$ before surgery and at 1 month after surgery, respectively (Fig. 3A); the OPG levels were $5.36 \pm 1.26$ and $9.47 \pm 2.28 \mathrm{ng} / \mathrm{ml}$ before and at 1 month after surgery, respectively (Fig. 3B). The levels of serum RANKL and OPG in the research group at 1 month after surgery were significantly higher than those before surgery $(\mathrm{P}<0.001)$ (Fig. 3A and B). Pearson's correlation analysis revealed that the levels of serum miR-142 and BMP-2 positively correlated with the levels of RANKL and OPG before surgery and at 1 month after surgery $(\mathrm{P}<0.001)$ (Fig. 3C-J).

\section{Discussion}

In recent years, the total number of hip fractures has markedly increased, and the majority of these occur in Asia (23). Clinically, the effect of hip replacement is to relieve joint pain, restore and improve joint mobility. However, some patients will suffer from dislocation of prosthesis bone and aseptic loosening after surgery, which prevents hip joint function returning to normal levels $(24,25)$. Therefore, it is of utmost significance for the post-operative rehabilitation of patients to identify indicators related to the recovery after surgery and to intervene in time.

In recent years, miRNAs and fractures have received extensive attention $(26,27)$. In the study by Liu et al (28), miR-137 was shown to prevent ALP activity and expression by inhibiting the expression of leucine-rich repeated $G$ protein-coupled receptor 4, making patients prone to osteoporosis fracture. In the study by Yao et al (29), miR-185 was shown to inhibit the growth and proliferation of osteoblasts during fracture healing by downregulating Wnt/ $\beta$-catenin axis targeting the PTH gene. These studies demonstrated that miRNAs play an important role in fractures. However, there are few studies available to date on the role of miR-142 in hip arthroplasty. BMP-2 is a classical morphogenetic hormone and a molecule that affects cell behavior. In mesenchymal cells, the level of BMP-2 affects myogenesis, lipogenesis, cartilage formation and bone formation (30). In the present study, the levels of serum miR-142 and BMP-2 in the pre-operative research group were significantly lower than those in the control group, and significantly increased at 1 month after surgery. There was a positive correlation between the serum miR-142 and BMP-2 levels both pre-operatively and post-operatively. The levels of serum miR-142 and BMP-2 in patients with femoral neck fracture were significantly lower than those in healthy individuals. 
Table III. ROC curve analysis of pre-operative serum miR-142 and BMP-2 in predicting the recovery of hip arthroplasty.

\begin{tabular}{lcccccc}
\hline Indicator & AUC & $95 \%$ CI & Std. Error & Cut-off & Sensitivity (\%) & Specificity (\%) \\
\hline miR-142 & 0.911 & $0.843-0.978$ & 0.034 & 1.81 & 95.65 & 88.89 \\
BMP-2 (ng/ml) & 0.861 & $0.789-0.933$ & 0.037 & 3.97 & 81.36 & 85.18 \\
\hline
\end{tabular}

A

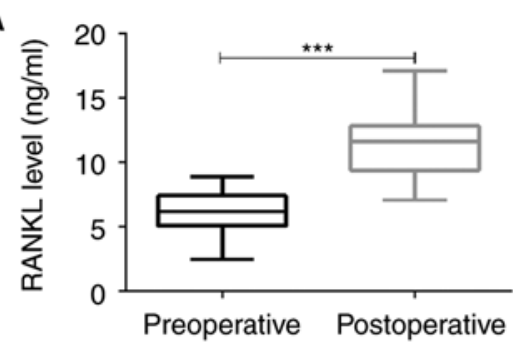

D

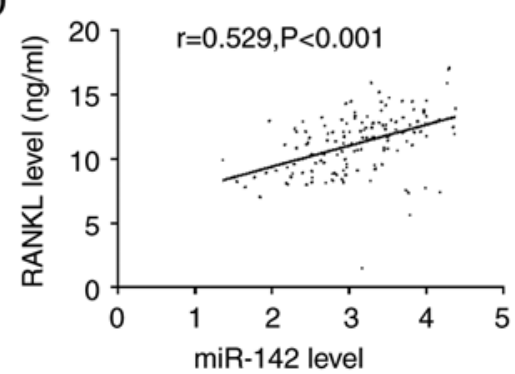

G

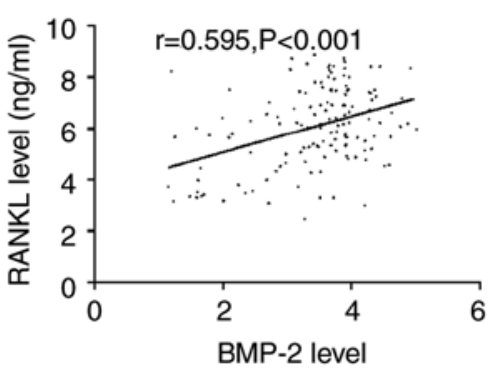

B
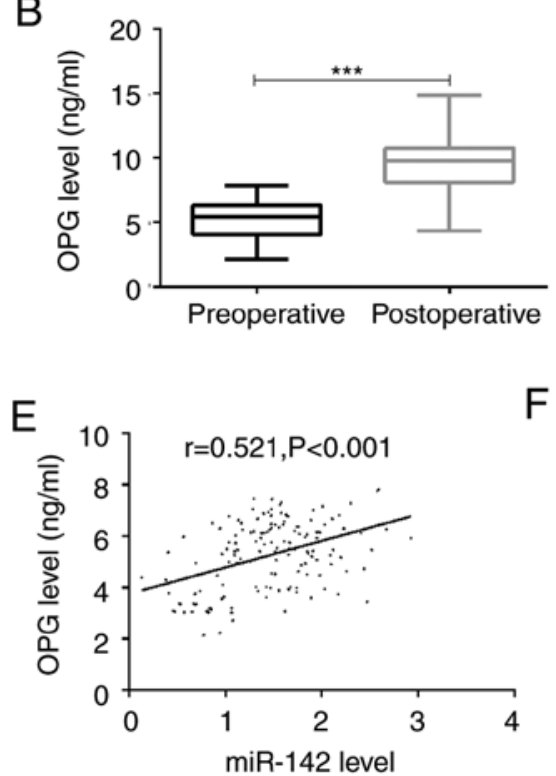

$\mathrm{H}$

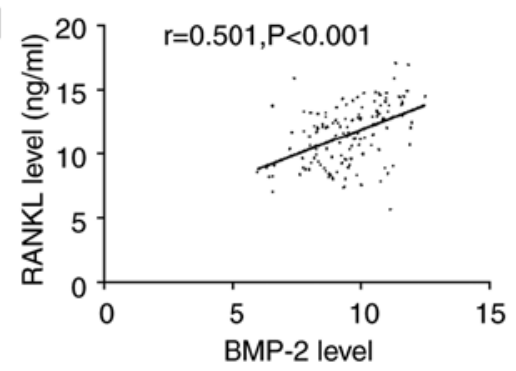

$\mathrm{F}$
C
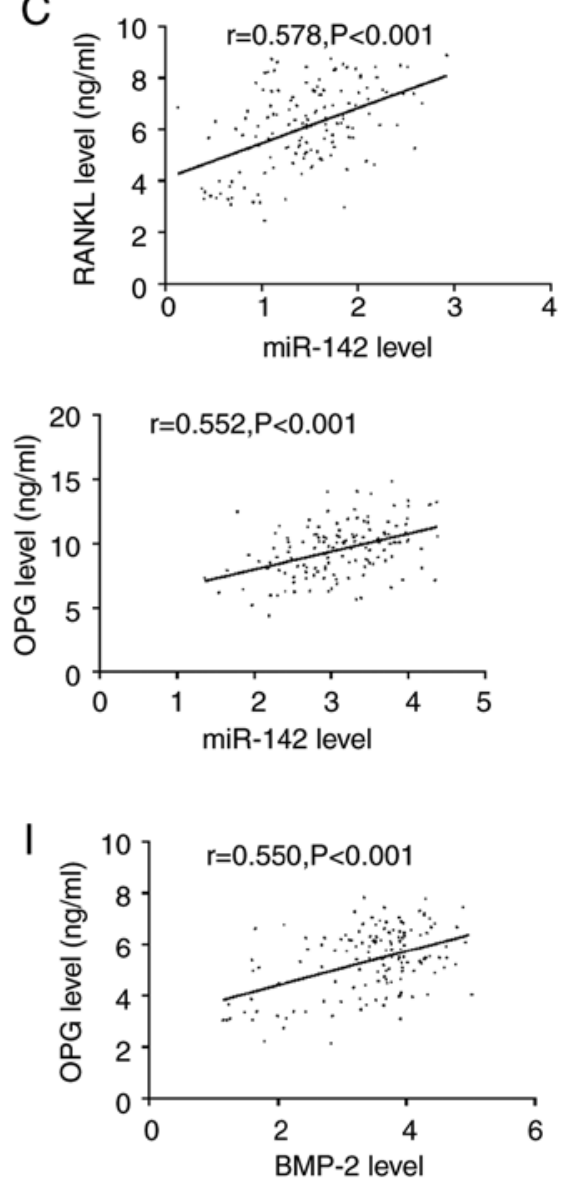

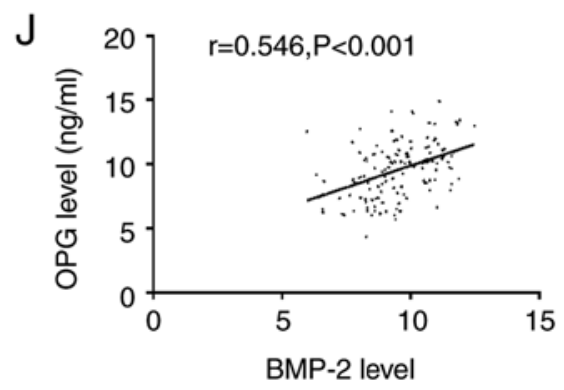

Figure 3. Correlation between serum RANKL and OPG levels before and at 1 month after surgery in the research group with miR-142 and BMP-2. The serum (A) RANKL and (B) OPG level at 1 month after surgery was significantly higher than that before surgery. There was a positive correlation between miR-142 and RANKL (C) before surgery and (D) after surgery. There was a positive correlation between miR-142 and OPG (E) before surgery and (F) after surgery. There was a positive correlation between miR-142 and RANKL (G) before surgery and (H) after surgery. Post-operative BMP-2 levels positively correlated with those of (I) RANKL and (J) OPG, respectively. ${ }^{* * *} \mathrm{P}<0.001$. BMP-2, bone morphogenetic protein-2; RANKL, receptor activator of NF- $\kappa \mathrm{B}$ ligand; OPG, osteoprotegerin.

The levels of serum miR-142 and BMP-2 in patients following hip replacement were significantly increased. In the study by Zhao et al (31), the level of miR-142-5p in older mice was lower than that in young mice, and was closely related to the age-related bone healing delay, while miR-142-5p was significantly upregulated and related to the generation of active osteoblasts during bone healing. However, Tsuji et al (32) considered that BMP-2 was an essential component of fracture 
repair. In bones lacking BMP-2, the earliest steps of fracture healing seemed to be blocked, and BMP-2 was identified as a necessary endogenous medium for fracture repair. This may be that following a fracture, the production of miR-142 and BMP-2 decreased, and miR-142 and BMP-2 played a similar role as fracture repair media in the recovery process; however, the mechanisms involved remain unclear. It was hypothesized that miR-142 and BMP-2 may be involved in the healing process of bone formation following hip replacement surgery. The Harris score is a standard for the curative effect of artificial total hip joint, which can reflect the recovery of hip joint function of patients and evaluate the therapeutic effect of hip joint replacement (33). The results of this study showed that preoperative serum miR-142 and BMP-2 levels had certain predictive value for the postoperative recovery of patients. As hip replacement surgery is a hip joint repair surgery for patients with fractures (34), the joint function of the patients was markedly improved, and the levels of serum miR-142 and BMP-2 were markedly increased at 1 month after surgery; thus, the fracture site may be at the recovery stage at this time. In addition, the levels of serum miR-142 and BMP-2 in patients with good recovery were significantly higher than those in patients with poor recovery at 1 month after surgery, and the serum miR-142 and BMP-2 levels positively correlated with the recovery effect. Therefore, the observation of miR-142 and BMP-2 has evaluating value for the recovery of patients following hip replacement surgery. In the study by de Amorim et al (35), the decrease in the number of RANKL- and OPG-positive cells in the tissues of diabetic rats following tibial fracture was significantly lower than that of healthy rats, and the number of RANKL- and OPG-positive cells was still significantly lower than that of the control group at 14 days. However, in the study by Wang et al (36), the levels of RANKL and OPG were significantly increased at 4 weeks after surgery for fracture patients, which was closely related to the fracture healing process. In the present study, the levels of serum RANKL and OPG in the research group were lower than those in the control group before surgery, but increased significantly at 1 month after surgery. The levels of miR-142 and BMP-2 positively correlated with the levels of RANKL and OPG before and after surgery. miR-142 and BMP-2 may thus participate in bone formation and bone metabolism, although the underlying mechanisms remain to be further explored.

Of course, the present study has certain limitations. Firstly, the present study did not observe the complications of patients following hip replacement surgery. Secondly, the regulatory mechanisms of miR-142 and BMP-2 in hip replacement surgery for patients with femoral neck fracture were not observed. These deficiencies need to be further attended to in future research.

In conclusion, the present study demonstrates that the pre-operative serum miR-142 and BMP-2 levels are valuable for evaluating the recovery of patients with femoral neck fracture following hip replacement surgery. In addition, at 1 month after surgery, the levels of both miR-142 and BMP-2 were related to the recovery of the patients, and positively correlated with the RANKL and OPG levels.

\section{Acknowledgements}

Not applicable.

\section{Funding}

No funding was received.

\section{Availability of data and materials}

The datasets used and/or analyzed during the current study are available from the corresponding author on reasonable request.

\section{Authors' contributions}

HG wrote the manuscript, and analyzed and interpreted the patient data. XW performed PCR and ELISA, and assisted with the statistical analysis. Both authors read and approved the final manuscript.

\section{Ethics approval and consent to participate}

The present study was approved by the Ethics Committee of The Fourth Hospital of Jinan. Patients who participated in this research, signed informed consent forms and had complete clinical data. Signed written informed consents were obtained from the patients and/or guardians.

\section{Patient consent for publication}

Not applicable.

\section{Competing interests}

The authors declare that they have no competing interests.

\section{References}

1. Yu L, Wang Y and Chen J: Total hip arthroplasty versus hemiarthroplasty for displaced femoral neck fractures: Meta-analysis of randomized trials. Clin Orthop Relat Res 470: 2235-2243, 2012.

2. Lowe JA, Crist BD, Bhandari M and Ferguson TA: Optimal treatment of femoral neck fractures according to patient's physiologic age: An evidence-based review. Orthop Clin North Am 41: 157-166, 2010.

3. Miyamoto RG, Kaplan KM, Levine BR, Egol KA and Zuckerman JD: Surgical management of hip fractures: An evidence-based review of the literature. II: Intertrochanteric fractures. J Am Acad Orthop Surg 16: 596-607, 2008.

4. Alnahhal A, Aslam-Pervez N and Sheikh HQ: Templating Hip arthroplasty. Open Access Maced J Med Sci 7: 672, 2019.

5. Nemes S, Lind D, Cnudde P, Bülow E, Rolfson O and Rogmark C: Relative survival following hemi-and total hip arthroplasty for hip fractures in Sweden. BMC Musculoskelet Disord 19: 407, 2018.

6. Maratt JD, Gagnier JJ, Butler PD, Hallstrom BR, Urquhart AG and Roberts KC: No difference in dislocation seen in anterior vs posterior approach total hip arthroplasty. J Arthroplasty 31: 127-130, 2016.

7. Li D, Liu J, Guo B, Liang C, Dang L, Lu C, He X, Cheung HY, $\mathrm{Xu} \mathrm{L}, \mathrm{Lu} \mathrm{C}$, et al: Osteoclast-derived exosomal miR-214-3p inhibits osteoblastic bone formation. Nat Commun 7: 10872, 2016.

8. Lian JB, Stein GS, van Wijnen AJ, Stein JL, Hassan MQ, Gaur T and Zhang Y: MicroRNA control of bone formation and homeostasis. Nat Rev Endocrinol 8: 212, 2012.

9. Xu R, Bi C, Song J, Wang L, Ge C, Liu X and Zhang M: Upregulation of miR-142-5p in atherosclerotic plaques and regulation of oxidized low-density lipoprotein-induced apoptosis in macrophages. Mol Med Rep 11: 3229-3234, 2015. 
10. Schaefer JS, Montufar-Solis D, Vigneswaran N and Klein JR: Selective upregulation of microRNA expression in peripheral blood leukocytes in IL-10-/- mice precedes expression in the colon. J Immunol 187: 5834-5841, 2011.

11. Zhang X, Yan Z, Zhang J, Gong L, Li W, Cui J, Liu Y, Gao Z, Li J, Shen L and Lu Y: Combination of hsa-miR-375 and hsa-miR-142-5p as a predictor for recurrence risk in gastric cancer patients following surgical resection. Ann Oncol 22: 2257-2266, 2011.

12. Wang YK, Yu X, Cohen DM, Wozniak MA, Yang MT, Gao L, Eyckmans J and Chen CS: Bone morphogenetic protein-2-induced signaling and osteogenesis is regulated by cell shape, RhoA/ROCK, and cytoskeletal tension. Stem Cells Dev 21: 1176-1186, 2011.

13. Chen J, Li K, Pang Q, Yang C, Zhang H, Wu F, Cao H, Liu H, Wan Y, Xia W, et al: Identification of suitable reference gene and biomarkers of serum miRNAs for osteoporosis. Sci Rep 6: 36347, 2016.

14. Mi M, Jin H, Wang B, Yukata K, Sheu TJ, Ke QH, Tong P, Im HJ, Xiao G and Chen D: Chondrocyte BMP2 signaling plays an essential role in bone fracture healing. Gene 512: 211-218, 2013.

15. Krege JH, Lane NE, Harris JM and Miller PD: PINP as a biological response marker during teriparatide treatment for osteoporosis. Osteoporos Int 25: 2159-2171, 2014.

16. Martin TJ and Sims NA: RANKL/OPG; Critical role in bone physiology. Rev Endocr Metab Disord 16: 131-139, 2015.

17. Cassuto J, Folestad A, Göthlin J, Malchau H and Kärrholm J: The key role of proinflammatory cytokines, matrix proteins, RANKL/OPG and Wnt/ $\beta$-catenin in bone healing of hip arthroplasty patients. Bone 107: 66-77, 2018.

18. Davenport DL, Bowe EA, Henderson WG, Khuri SF and Mentzer RM Jr: National surgical quality improvement program (NSQIP) risk factors can be used to validate American Society of Anesthesiologists Physical Status Classification (ASA PS) levels. Ann Surg 243: 636-644, 2006.

19. Wright NC, Saag KG, Dawson-Hughes B, Khosla S and Siris ES The impact of the new National Bone Health Alliance (NBHA) diagnostic criteria on the prevalence of osteoporosis in the USA. Osteoporos Int 28: 1225-1232, 2017.

20. Sprague S, Bhandari M, Heetveld MJ, Liew S, Scott T, Bzovsky S, Heels-Ansdell D, Zhou Q, Swiontkowski M and Schemitsch EH FAITH Investigators: Factors associated with health-related quality of life, hip function, and health utility after operative management of femoral neck fractures. Bone Joint J 100-B: 361-369, 2018.

21. Singh JA, Schleck C, Harmsen S and Lewallen D: Clinically important improvement thresholds for Harris Hip Score and its ability to predict revision risk after primary total hip arthroplasty. BMC Musculoskelet Disord 17: 256, 2016.

22. Livak KJ and Schmittgen TD: Analysis of relative gene expression data using real-time quantitative PCR and the 2(-Delta Delta C(T)) method. Methods 25: 402-408, 2001.

23. Abdel MP, Watts CD, Houdek MT, Lewallen DG and Berry DJ: Epidemiology of periprosthetic fracture of the femur in 32644 primary total hip arthroplasties: A 40-year experience. Bone Joint J 98: 461-467, 2016.
24. Mahmood SS, Mukka SS, Crnalic S, Wretenberg P and Sayed-Noor AS: Association between changes in global femoral offset after total hip arthroplasty and function, quality of life, and abductor muscle strength: A prospective cohort study of 222 patients. Acta Orthop 87: 36-41, 2016.

25. Angerame MR, Fehring TK, Masonis JL, Mason JB, Odum SM and Springer BD: Early failure of primary total hip arthroplasty: Is surgical approach a risk factor? J Arthroplasty 33: 1780-1785, 2018.

26. Lee WY, Li N, Lin S, Wang B, Lan HY and Li G: miRNA-29b improves bone healing in mouse fracture model. Mol Cell Endocrinol 430: 97-107, 2016.

27. Waki T, Lee SY, Niikura T, Iwakura T, Dogaki Y, Okumachi E, Oe K, Kuroda R and Kurosaka M: Profiling microRNA expression during fracture healing. BMC Musculoskelet Disord 17: 83, 2016.

28. Liu X and Xu X: MicroRNA-137 dysregulation predisposes to osteoporotic fracture by impeding ALP activity and expression via suppression of leucine-rich repeat-containing G-protein-coupled receptor 4 expression. Int J Mol Med 42: 1026-1033, 2018.

29. Yao CJ, Lv Y, Zhang CJ, Jin JX, Xu LH, Jiang J, Geng B, Li H, Xia YY and Wu M: MicroRNA-185 inhibits the growth and proliferation of osteoblasts in fracture healing by targeting PTH gene through down-regulating Wnt/ $\beta$-catenin axis: In an animal experiment. Biochem Biophys Res Commun 501: 55-63, 2018.

30. Rogers MB, Shah TA and Shaikh NN: Turning bone morphogenetic protein 2 (BMP2) on and off in mesenchymal cells. J Cell Biochem 116: 2127-2138, 2015.

31. Zhao R, Zhu Y and Sun B: Exploration of the Effect of mmu-miR-142-5p on Osteoblast and the Mechanism. Cell Biochem Biophys 71: 255-260, 2015.

32. Tsuji K, Bandyopadhyay A, Harfe BD, Cox K, Kakar S, Gerstenfeld L, Einhorn T, Tabin CJ and Rosen V: BMP2 activity, although dispensable for bone formation, is required for the initiation of fracture healing. Nat Genet 38: 1424, 2006.

33. Singh JA, Schelck C, Harmsen S and Lewallen D: AB0757 Clinically important improvement thresholds for Harris hip score and its ability to predict revision risk after primary total hip arthroplasty. BMC Musculoskelet Disord 17: 256, 2016.

34. Higgins BT, Barlow DR, Heagerty NE and Lin TJ: Anterior vs. posterior approach for total hip arthroplasty, a systematic review and meta-analysis. J Arthroplasty 30: 419-434, 2015.

35. de Amorim FP, Ornelas SS, Diniz SF, Batista AC and da Silva TA Imbalance of RANK, RANKL and OPG expression during tibial fracture repair in diabetic rats. J Mol Histol 39: 401, 2008.

36. Wang XF, Zhang YK, Yu ZS and Zhou JL: The role of the serum RANKL/OPG ratio in the healing of intertrochanteric fractures in elderly patients. Mol Med Rep 7: 1169-1172, 2013.

This work is licensed under a Creative Commons Attribution-NonCommercial-NoDerivatives 4.0 International (CC BY-NC-ND 4.0) License. 